\begin{tabular}{|c|l|}
\hline Title & Lattice vibrational spectrum and possible existence of local ized modes of the two-dimensional Penrose lattice \\
\hline Author(s) & Nishiguchi, Norihiko; Sakuma, Tetsuro \\
\hline Citation & $\begin{array}{l}\text { Physical Review B, 38(11), 7370.7377 } \\
\text { https://doi.org/10.1103/PhysRevB.38.7370 }\end{array}$ \\
\hline Issue Date & 1988-10-15 \\
\hline Doc URL & http://hdl.handle.net/2115/47087 \\
\hline Rights & ○ 1988 The A merican Physical Society \\
\hline Type & article \\
\hline File Information & PhysRevB.38.7370.pdf \\
\hline
\end{tabular}

Instructions for use 


\title{
Lattice vibrational spectrum and possible existence of localized modes of the two-dimensional Penrose lattice
}

\author{
Norihiko Nishiguchi and Tetsuro Sakuma \\ Department of Engineering Science, Hokkaido University, Sapporo 060, Japan
}

(Received 1 February 1988)

\begin{abstract}
Lattice vibrations on two-dimensional Penrose lattices are investigated numerically. The resultant spectra exhibit several peaks similar to those of disordered systems. From the dependence of the vibrational phase difference and the distribution of the local elastic energy on the different boundary conditions, it is found that the lattice vibrations of this system seem to be isotropic and localized above a certain critical frequency. The spectral peaks are attributed to van Hove singularities and localization.
\end{abstract}

\section{INTRODUCTION}

Since the recent discovery of quasicrystalline phases in a rapidly quenched alloy of $\mathrm{Al}$ with $\mathrm{Mn}$ by Schechtman et al., 1 there has been great interest in the physics of quasicrystals. Although quasicrystals do not have periodicity, there is still a long-range bond orientational order in the structure. Therefore quasicrystals are quite different ordered states from crystals and amorphous solids. ${ }^{2}$ Such a characteristic structure of the system will lead to new features in the lattice vibrational properties as well as the thernıal properties of quasicrystals. Lattice vibrations of quasiperiodic systems have been studied theoretically. $^{3-10}$ It has been shown that in the onedimensional quasicrystal or the Fibonacci chain, there are spectral gaps due to the quasiperiodic structure and that the spatial variations of the eigenfunctions are intermediate between localized and extended in the highfrequency region. ${ }^{3}$

For icosahedral quasicrystals, phonons and phasons have been well discussed based on spatial symmetries as hydrodynamic elastic modes with long wavelengths. ${ }^{4-7}$ Anisotropic phonon attenuation due to the characteristic structural order is found while the sound velocities are isotropic. $^{5}$ The lattice vibrational properties of twodimensional quasicrystals have been studied numerically by several authors. ${ }^{8-10}$ Lattice vibrations of the Penrose lattice perpendicular to the lattice plane were investigated by Odagaki and Nguyen ${ }^{8}$ who found energy gaps in the spectrum due to the nonperiodic structure. They also declared the peaks in the vibrational modes around the gaps to be van Hove singularities. Kohmoto and Sutherland, ${ }^{9}$ on the other hand, suggested the existence of the localized modes such as the confined electronic states with zero energy in a system similar to that of Odagaki et al.

The purpose of this paper is to investigate the characteristic features in the structure of the lattice vibrational spectrum of quasicrystals. In this paper, we take twodimensional Penrose tilings consisting of a pair of rhom$\mathrm{bi}^{11}$ as the two-dimensional quasicrystal. We also take into account displacements parallel to the Penrose lattice.
The Penrose tilings are obtained by the inflation method $^{12}$ up to the fifth generations from a seed with five fat rhombuses. There are two types of the figures due to the manner of inflations distinguished by the central figure. We show in Fig. 1 one of the Penrose tilings for the fifth generation. In the following sections, to avoid confusion, we discuss the type in Fig. 1, since the characteristic results are independent of the structures of the two different Penrose tilings. The Penrose lattice is now defined as the atom-decorating Penrose tiling at each vertex of the rhombus.

In the following section, we specify the model of the two-dimensional vibrational system and formulate the dynamical matrix elements. In Sec. III the numerical results of the vibrational spectra, participation ratio, and vibrational phase are shown. By using these quantities, the characteristic features of the spectrum are investigated. A summary and discussion are presented in the last section.

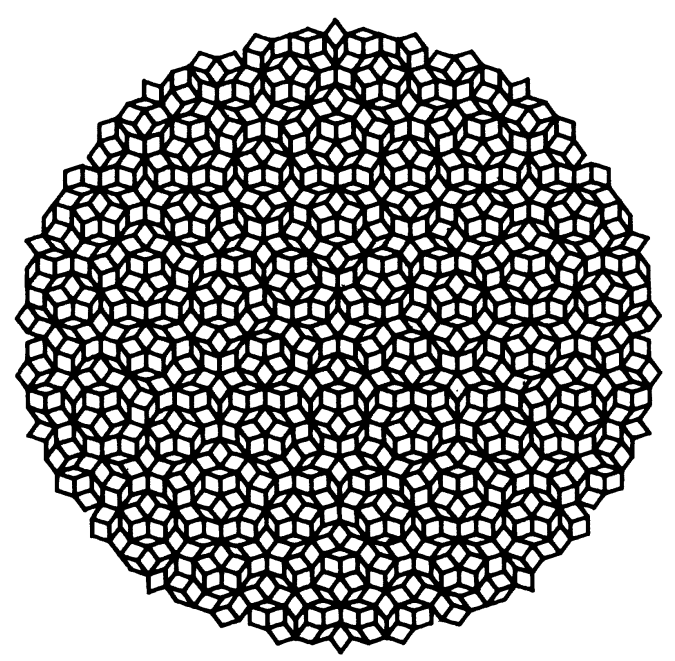

FIG. 1. Penrose tiling with 1231 sites for the fifth generation. The central figure is of a star. 


\section{LATTICE DYNAMICS OF PENROSE LATTICE}

In this section we present the model for the lattice vibrations of the two-dimensional Penrose lattice. We consider atomic vibrations in the plane parallel to the Penrose lattice and deal with vibrations in the harmonic approximation. We can therefore employ two independent restoring forces; one is proportional to the bond-length stretching and the other to the change in rhombus angle due to bond bending. To develop further, we devote ourselves to the deformation of one rhombus in the Penrose lattice as shown in Fig. 2. Consider the rhombus deformed under stress and let $\mathbf{P}_{i}^{0}$ and $\mathbf{u}_{i}$ be the lattice-site vector and the displacement vector of the atom at the $i$ th site, respectively. Then the position vector of the atom at the $i$ th site after deformation is given by $\mathbf{P}_{i}-\mathbf{P}_{i}^{0}+\mathbf{u}_{i}$. The relative lattice-site vector between the $j$ th site and the $i$ th one, $\mathbf{R}_{i j}^{0}$, is defined by $\mathbf{R}_{i j}^{0}=\mathbf{P}_{j}^{0}-\mathbf{P}_{i}^{0}$. In the same way, the relative position vectors $\mathbf{R}_{i j}$ between the $j$ th and $i$ th atoms are given by $\mathbf{R}_{i j}=\mathbf{P}_{j}-\mathbf{P}_{i}$. The difference in displacement vector $\mathbf{u}_{i j}$ between them can be obtained in terms of relative position vectors, which yields

$$
\begin{aligned}
\mathbf{u}_{i j} & =\mathbf{R}_{i j}-\mathbf{R}_{i j}^{0} \\
& =\mathbf{P}_{j}-\mathbf{P}_{i}-\left(\mathbf{P}_{j}^{0}-\mathbf{P}_{i}^{0}\right) \\
& =\mathbf{u}_{j}-\mathbf{u}_{i} .
\end{aligned}
$$

This relative displacement vector gives the stretching and rotation of the edges. The extension is expressed by the scalar product $\mathbf{u}_{i j} \cdot \boldsymbol{\epsilon}_{i j}$, where $\boldsymbol{\epsilon}_{i j}=\mathbf{R}_{i j}^{0} /\left|\mathbf{R}_{i j}^{0}\right|$. Here we take the restoring force to be proportional to the stretching of the edges and refer to it as the central force because it depends only on the stretching of bonds between atoms.

On the other hand, the vector product $\mathbf{u}_{i j} \times \epsilon_{i j}$ gives the change of the direction of the relative lattice-site vector $\mathbf{R}_{i j}^{0}$ to $\mathbf{R}_{i j}$ or the rotation of the $j$ th atom around the $i$ th atom. The opposite side edge that has the same end, $i$ th site, rotates in the same way, too. The difference between the angles, $\mathbf{u}_{i j} \times \epsilon_{i j}-\mathbf{u}_{i k} \times \epsilon_{i k}$, gives bond bending around the $i$ th site. We assume that the bending $\mathbf{u}_{i j} \times \epsilon_{i j}-\mathbf{u}_{i k} \times \epsilon_{i k}$ provides the torque around the $i$ th atom. The force acting on the $j$ th atom is derived by dividing the torque by the edge length. The direction is perpendicular to the edge. As a reaction, the $i$ th atom is pushed by the sum of the forces. There are torques around the other sites in the Penrose lattice in the same way. Then the ith atom is affected by the torque around the nearest-neighbor atoms. That is, each atom is affected not only by the nearest neighbors but also by the second nearest neighbors to maintain the lattice structure. We refer to it as the angular force, since it is due to bond bending.

The coordination number of sites in the Penrose lattice has a value from 2 to 7 as seen in Fig. 1. Therefore it is too complicated to construct the dynamical matrix elements individually for each vertex in the way mentioned above. Instead we introduce the harmonic potential ener-

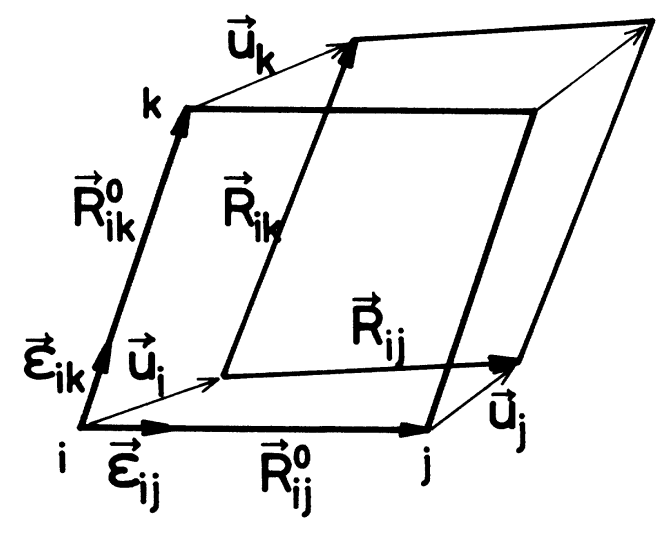

FIG. 2. Relative position vectors between lattice sites, $\mathbf{R}_{i j}^{0}$, displacement vectors of atoms, $\mathbf{u}_{i}$, and the relative position vectors between atoms, $\mathbf{R}_{i j}$, in a rhombus.

gy to derive the dynamical matrix elements systematically.

The potential energy consists of two terms: one is due to the bond stretching and the other due to the bond bending of rhombuses,

$$
V=V_{c}+V_{a}
$$

where

$$
\begin{aligned}
& V_{c}=\frac{k}{2} \sum_{i, j}\left[\left(\mathbf{u}_{j}-\mathbf{u}_{i}\right) \cdot \epsilon_{i j}\right]^{2}, \\
& V_{a}=\frac{g}{2} \sum_{i, j, k}\left(\theta_{i j}-\theta_{i k}\right)^{2}
\end{aligned}
$$

where $\boldsymbol{\theta}_{i j}$ is the rotation angle of the $j$ th atom around the $i$ th atom defined by $\boldsymbol{\theta}_{i j}=\epsilon_{i j} \times \mathbf{u}_{i j} /\left|\mathbf{R}_{i j}^{0}\right|$. The summation in the first term is performed over all the connected pairs. The sum in the second term is over all the angles of the rhombuses in the lattice. $k$ and $g$ are the force constants of the central and the angular forces, respectively.

The dynamical matrix elements $C_{l n}^{\alpha \beta}$ due to the bond stretching can be obtained by the partial derivative of the potential with respect to the displacement vector as

$$
C_{l n}^{\alpha \beta}=\delta_{l, n} k \sum_{i(\neq l)} \epsilon_{i l}^{\alpha} \epsilon_{i l}^{\beta}-k \epsilon_{n l}^{\alpha} \epsilon_{n l}^{\beta}\left(1-\delta_{l, n}\right) .
$$

Here the superscripts $\alpha$ and $\beta$ denote the Cartesian coordinates. The potential energy due to the bond bending is rewritten in terms of the displacement vectors as follows:

$V_{a}=\frac{g}{2} \sum_{i, j, k}\left(\epsilon_{i j} \times \mathbf{u}_{j}-\epsilon_{i j} \times \mathbf{u}_{i}-\epsilon_{i k} \times \mathbf{u}_{k}+\epsilon_{i k} \times \mathbf{u}_{i}\right)^{2}$.

The dynamical matrix elements $A_{l n}^{\alpha \beta}$, due to the bond bending is similarly obtained as 


$$
\begin{aligned}
& A_{l n}^{\alpha \beta}=-\frac{g}{2} \sum_{j, k}\left[\left(\epsilon_{l k}^{\alpha}-\epsilon_{l j}^{\alpha}\right)\left(\epsilon_{l k}^{\beta}-\epsilon_{l j}^{\beta}\right)-\delta^{\alpha, \beta} \sum_{\gamma}\left(\epsilon_{l k}^{\gamma}-\epsilon_{l j}^{\gamma}\right)\left(\epsilon_{l k}^{\gamma}-\epsilon_{l j}^{\gamma}\right)\right]-g \sum_{k, m}\left[\epsilon_{k l}^{\alpha} \epsilon_{k l}^{\beta}-\delta^{\alpha, \beta} \sum_{\gamma} \epsilon_{k l}^{\gamma} \epsilon_{k l}^{\gamma}\right] \text { for } n=l, \\
& A_{l n}^{\alpha \beta}=-g \sum_{\gamma}\left[\epsilon_{n l}^{\alpha}\left(\epsilon_{l k}^{\beta}-\epsilon_{l n}^{\beta}\right)-\delta^{\alpha, \beta} \sum_{\gamma} \epsilon_{l n}^{\gamma}\left(\epsilon_{l k}^{\gamma}-\epsilon_{l n}^{\gamma}\right)\right]-g \sum_{k}\left[\epsilon_{n l}^{\beta}\left(\epsilon_{n k}^{\alpha}-\epsilon_{n l}^{\alpha}\right)-\delta^{\alpha, \beta} \sum_{\gamma} \epsilon_{n l}^{\gamma}\left(\epsilon_{n k}^{\gamma}-\epsilon_{n l}^{\gamma}\right)\right] \\
& A_{l n}^{\alpha \beta}=g \sum_{\gamma}\left[\epsilon_{j n}^{\alpha} \epsilon_{j l}^{\beta}-\delta^{\alpha, \beta} \sum_{\gamma} \epsilon_{j n}^{\gamma} \epsilon_{j l}^{\gamma}\right) \text { for } n=l+1 \text { or } n=l-1,
\end{aligned}
$$

The first equation, (2.6a), is for the diagonal elements. The summations over $j$ and $k$ are performed for the nearest neighbors of the $l$ th site and the sum over $m$ for the second nearest neighbors through the atom at the $k$ th site. The second one, $(2.6 \mathrm{~b})$, is for the case that the two sites are the nearest neighbors. We sum up the terms for the nearest neighbors of atoms at the $l$ th or the $n$th site. The last one, (2.6c), is for the second-nearest neighbors, in which the summation is carried over the nearest neighbors of both atoms at the $l$ th and the $n$th sites.

The equation of motion is written in terms of the dynamical matrix $\left\{D_{\ln }^{\alpha \beta}\right\}$ as follows:

$$
m \ddot{u}_{l}^{\alpha}-\sum_{n} \sum_{\beta} D_{l n}^{\alpha \beta} u_{n}^{\beta}=0,
$$

where $D_{l n}^{\alpha \beta}=C_{l n}^{\alpha \beta}+A_{l n}^{\alpha \beta}$. Here we assumed that all masses are unity. Therefore the vibrational properties of this system depend on the two independent force constants $k$ and $g$.

In the following section, we apply this equation to the Penrose lattices with different sizes and obtain eigenfrequencies and eigenfunctions numerically under different boundary conditions.

\section{VIBRATIONAL SPECTRA AND ENERGY DISTRIBUTION}

\section{A. Density of states: $D\left(\omega^{2}\right)$}

At first, as a reference, we calculate the density of states $D\left(\omega^{2}\right)$ of regular square lattice of 1225 atoms in a $35 \times 35$ array. The force constants $k$ and $g$ are assumed to be unity, respectively, and a free boundary condition is used. Results are shown in Fig. 3. The solid line exhibits the exact solution for the regular lattice which can be described in terms of the complete elliptic integral of the first kind. ${ }^{13}$ There occur van Hove singularities at $\omega^{2}=3.5$ and 16.0. The agreement with numerical results is excellent except the low-frequency region below $\omega^{2}=1.5$, where the lattice vibrations at the perimeter of the lattice are important. These vibrational modes are removed under a fixed boundary condition.

Next we apply the method to the Penrose lattice with two different sizes and plot the density of states in Fig. 4. Figure $4(\mathrm{a})$ is for the fourth generation with 481 atoms and Fig. 4(b) for the fifth one with 1231 atoms. The lowest three modes due to the uniform translations and rotation are excluded in these spectra. We can see clearly the difference in the density of states between the regular lattice and the Penrose one. In the former case, only two cusps are found due to the van Hove singularities. On the contrary, several peaks are found in the latter cases as seen in mass-disordered systems. ${ }^{14}$ The lowest peak appears at lower frequency than that of regular lattice and becomes moderate. The others spread out through the whole range of frequency. The dominant spectral peaks appear at $\omega^{2}=2.5,9.3,13.0,17.1,21.0$, and 24.0. There is a large gap around $\omega^{2}=24$ and some small gaps below it. Although the small gaps disappear with increasing size, both cases with different sizes have the peaks at the same frequencies, and the global structures of spectra are quite similar independent of the lattice size. Therefore we may be able to extrapolate the properties of the infinite systems from those of finite ones.

To check the dependence of the spectra on the force constants, we keep $k=1$ and change the strength of the angular force constant $g$ continuously from 0 to 2 . Figure 5 shows the dependence of the eigenvalues on $g$ for the second-generated Penrose lattice for legibility. The

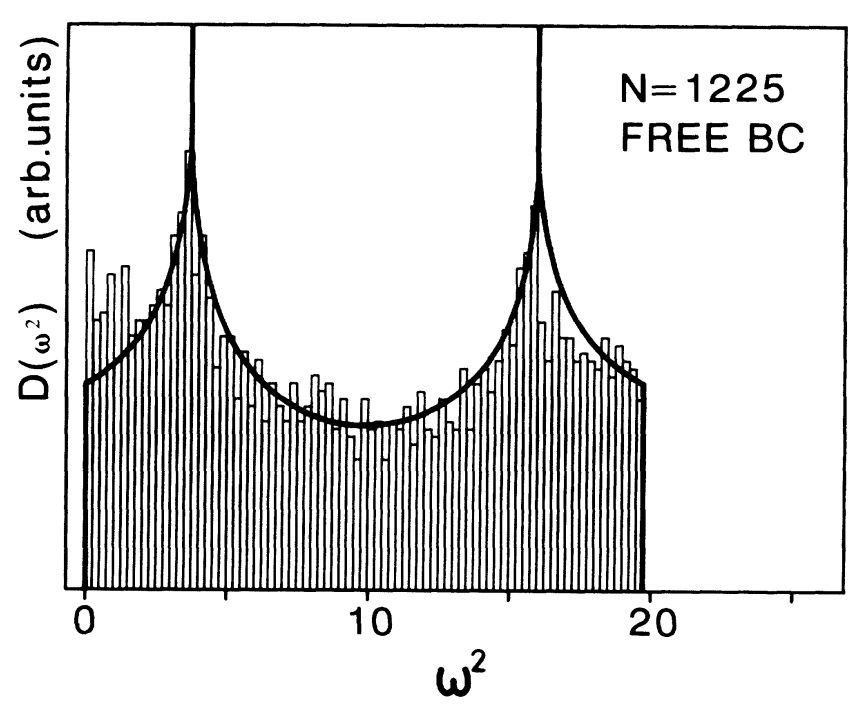

FIG. 3. Density of states of the lattice vibrations parallel to the $x-y$ plane for the two-dimensional square lattice with 1225 atoms in a $35 \times 35$ array under the free boundary condition. The solid line denotes the exact result described in terms of the complete elliptic integral of the first kind. The force constants $k$ and $g$ are taken to be unity, respectively. 
spectrum is sparse, and there are some large gaps that cannot be seen in Fig. 4. These gaps, however, vanish except the one with the highest frequency and the spectrum becomes denser in the larger systems because they are originated from size effect.

The distribution of the spectrum spreads as increasing $g$ and only a few modes cross above $g=0.5$. In the lowfrequency region, the eigenvalues are almost independent of the force constant $g$. In the high-frequency region, however, the eigenvalues seem to depend severely on the value of $g$. To see the difference in spectra more clearly, we calculate eigenfrequencies for the cases with $(k=1, g=1)$ and $(k=1, g=2)$ for the fourth generation and plot eigenfrequencies versus mode number for these
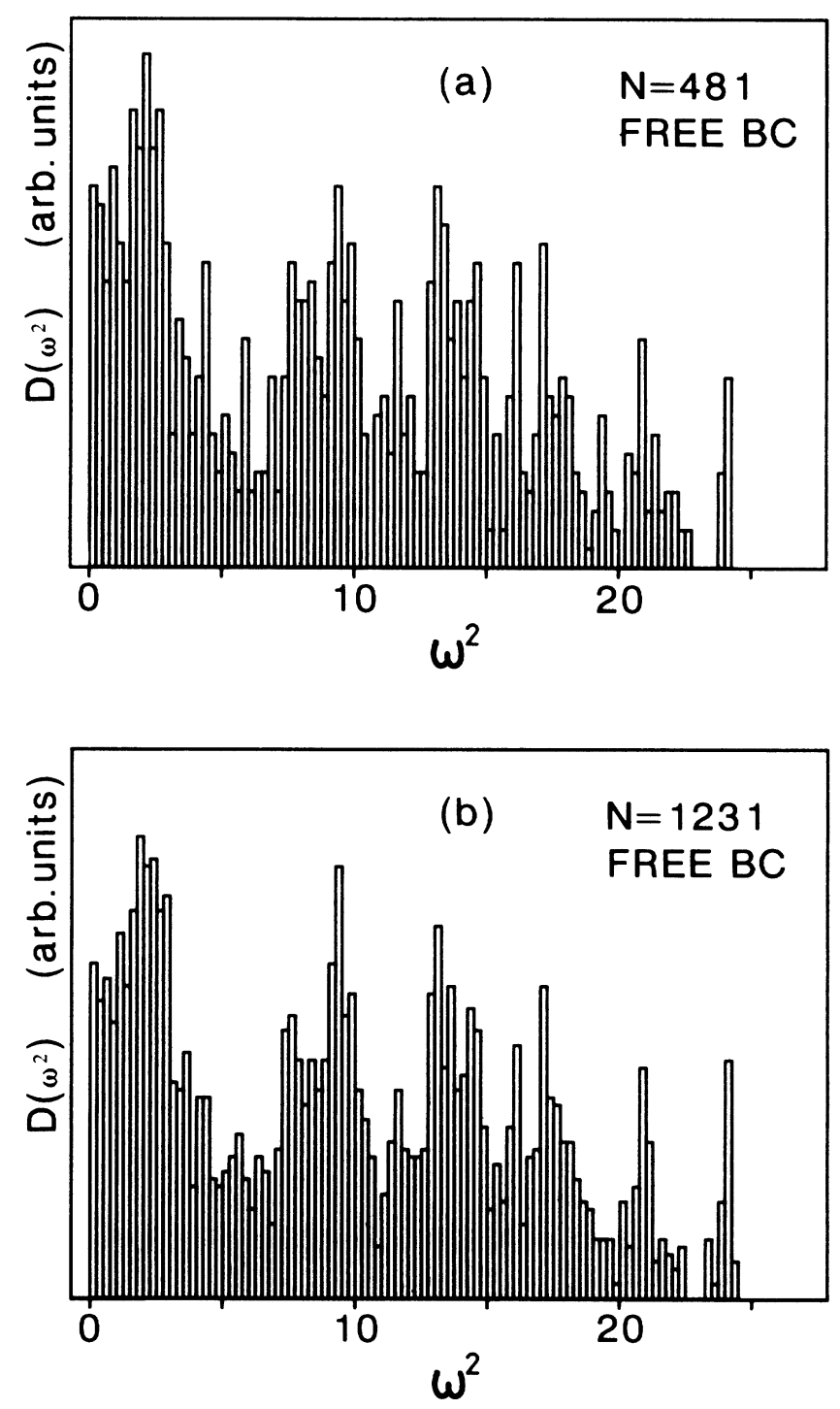

FIG. 4. Density of states of the lattice vibrations of the Penrose lattice (a) with 481 sites for the fourth generation under the free boundary condition. The structure is the same as Fig. 1. The displacement vectors are parallel to the lattice plane. (b) Penrose lattice with 1231 sites for the fifth generation.

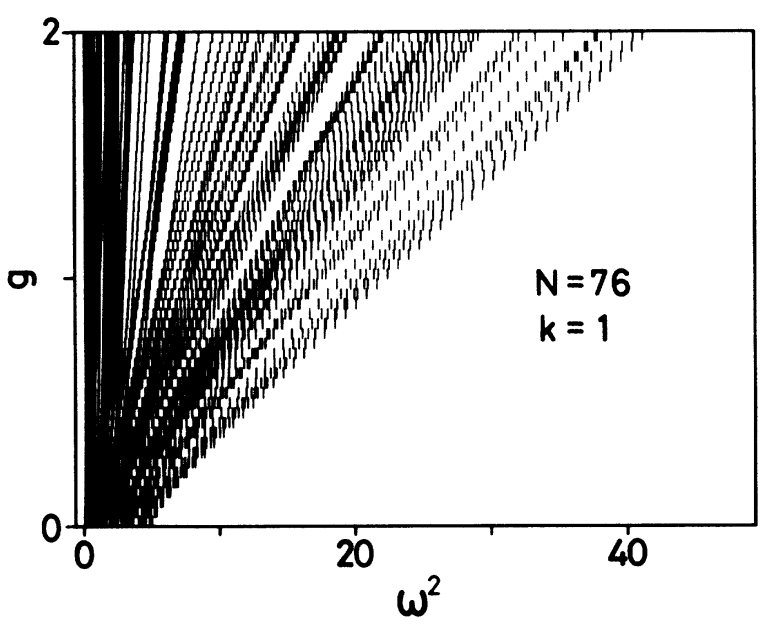

FIG. 5. Dependence of the lattice vibrational spectrum of the Penrose lattice with 76 sites for the second generation on force constant $g$. The force constant $k$ is kept to be unity.

two cases in Fig. 6. The difference in eigenfrequencies increases in the region of large mode number. However, the dependence of the eigenfrequencies on the mode number for both cases is quite similar and does not change drastically.

Then we conclude that the spectrum does not lose the essential characteristic features with changing force constants. We, therefore, investigate the vibrational properties with both $k$ and $g$ to be unity hereafter.

We have estimated the density of states so far to investigate how the bond-orientational order affects the lattice vibrations. The resultant density of states has the several peaks. Although we have supposed that the quasicrystals to be positioned between the regular periodic crystals and the disordered solids due to the long-range bond-

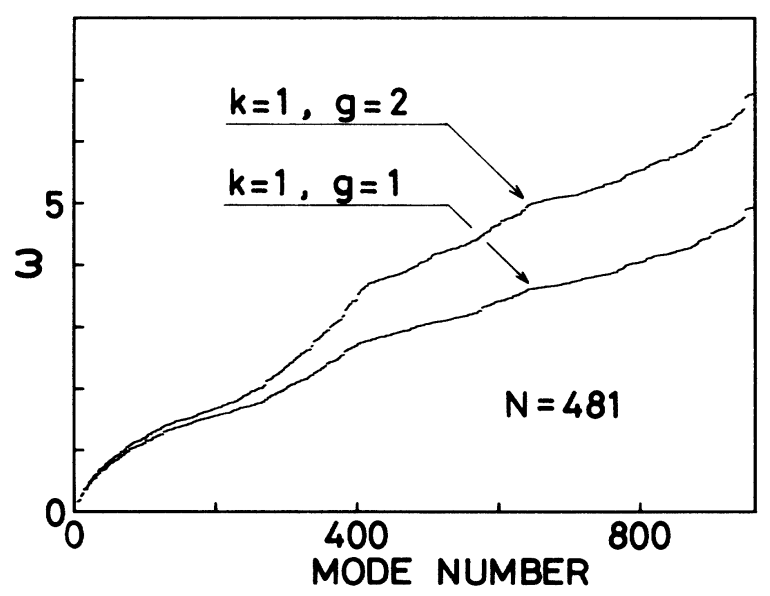

FIG. 6. Eigenfrequencies vs mode number of the Penrose lattice for the fourth generation. Two cases are plotted; one is for $(k=1, g=1)$ and the other for $(k=1, g=2)$. 
orientational order, our spectrum for the Penrose lattice seems to be rather similar to that of the mass-disordered lattices with light impurities ${ }^{14}$ than the regular ones. The spectral peaks in the mass-disordered system are due to the van Hove singularity and the localization. ${ }^{14}$ Here, if there are the localized vibrational modes in the Penrose lattice, such modes must be independent of the boundary conditions.

To investigate the dependence of the density of states on the boundary conditions, we calculate the density of states for the fifth generation under the fixed boundary condition. Figure 7 shows the resulted density of states. We see that the vibrational spectra do not depend on the boundary conditions in the whole frequency range except the low-frequency region $\omega^{2}<5.0$.

In the following subsections, the dependence of the lattice vibrations on the boundary conditions is investigated for the spatial distribution of elastic energy and also the vibrational phase.

\section{B. Energy distribution and participation ratio}

We consider the spatial distribution of local energy. The local energy $E\left(l, \omega_{\lambda}\right)$ is defined in terms of the calculated eigenvectors $\left\{\mathbf{u}_{i, \lambda}\right\}$ corresponding to the eigenfrequency $\omega_{\lambda}$ as follows

$$
E\left(l, \omega_{\lambda}\right)=\frac{1}{2}\left(\frac{1}{2} m \omega_{\lambda}^{2} \sum_{\alpha} u_{l, \lambda}^{\alpha} u_{l, \lambda}^{\alpha}+\frac{1}{2} \sum_{\alpha, \beta} u_{l, \lambda}^{\alpha} \sum_{m} D_{l m}^{\alpha \beta} u_{m, \lambda}^{\beta}\right)
$$

where $l$ denotes the site number index. The sum of the second term in Eq. (3.1) is over those atoms which are the nearest and the second nearest neighbors of the $l$ th site. The factor $\frac{1}{2}$ before the parenthesis is due to the time average. Here we introduce the participation ratio $P_{\lambda}\left(\omega_{\lambda}\right)$ defined by the ratio of square of averaged energy and the dispersion as follows: ${ }^{8}$

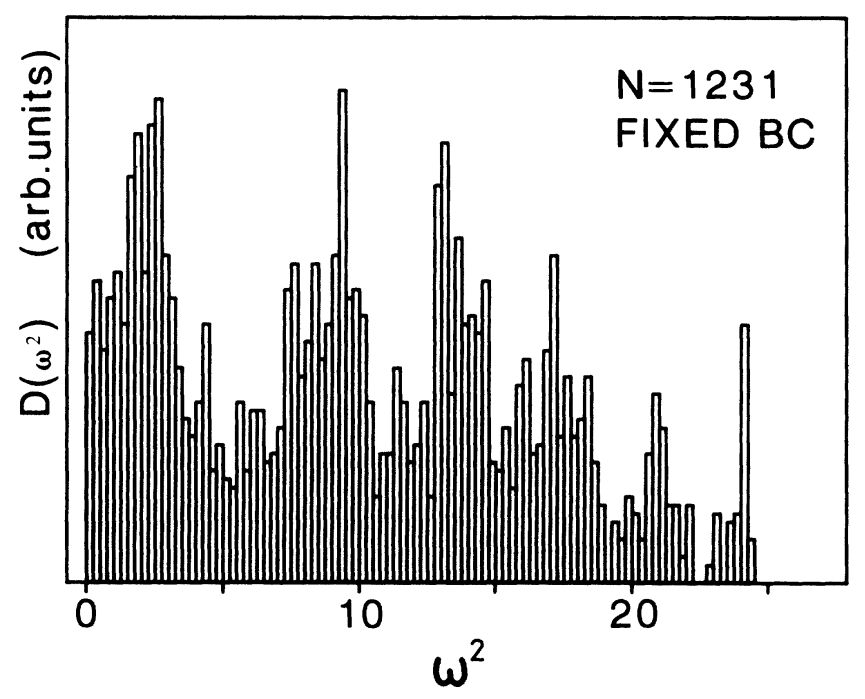

FIG. 7. Density of states of the lattice vibrations of the Penrose lattice with 1231 sites for the fifth generation with fixed boundary condition.

$$
P_{\lambda}\left(\omega_{\lambda}\right)=\left\langle E\left(l, \omega_{\lambda}\right)\right\rangle^{2} /\left\langle E\left(l, \omega_{\lambda}\right)^{2}\right\rangle .
$$

The angle brackets denote the statistical average over atoms. The participation ratio has the value of $1 / N$ when the energy concentrates on one atom, where $N$ is the total number of atoms. On the other hand, the $P_{\lambda}$ has the value of unity when the energy is shared among all atoms equally.

Figure 8(a) depicts the participation ratios of the Penrose lattice for the fourth generation with free boundary. The participation ratios are smaller than those of the regular lattice, which are about 0.6 through the frequency region and distribute widely below $P_{\lambda}=0.5$. The decrease of the values above $\omega^{2}=14$ also reveals the relevant feature. This means the energy concentrations in real space and may lead to the possible existence of the localization in the high-frequency region. The difference in the $P_{\lambda}$ from the distinct boundary condition, as seen in Fig. 8(b), appears apparently in the frequency region below $\omega^{2}=5$. The difference in the low-frequency region
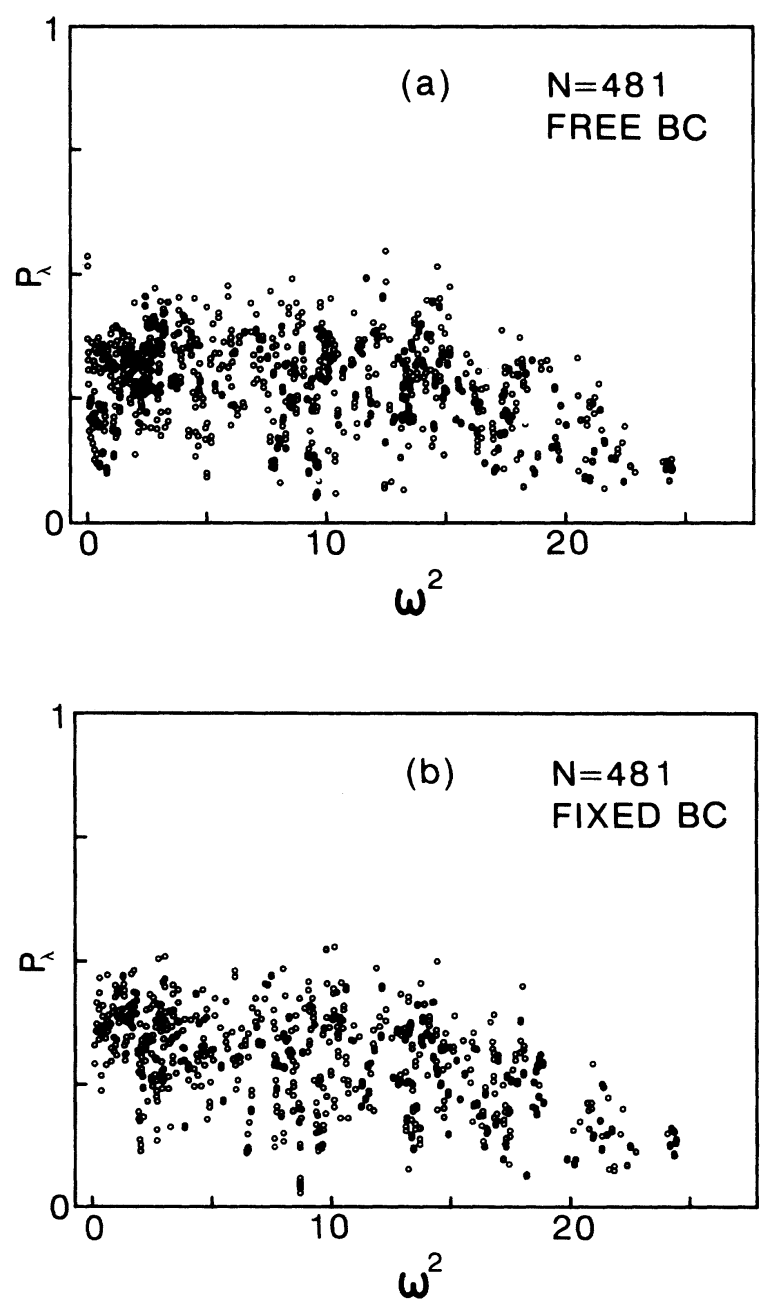

FIG. 8. The participation ratio $P_{\lambda}$ of the fourth-generated Penrose lattice. (a) Free boundary condition and (b) fixed boundary condition. 
is caused by the size effect that the lattice vibrations of the atoms at perimeter of the finite lattice are suppressed under the fixed boundary condition. However, above the region, noticeable changes in magnitude are not found between them as seen in the regular lattices.

\section{The vibrational phase shift due to boundary condition}

It is important to check the phase shift due to the change of the boundary conditions when we discuss the localization of lattice vibrations. ${ }^{15}$ Although the phase shift is expressed in terms of the wave vector and the corresponding frequency in the regular lattice, ${ }^{15}$ we have to take another parameter to describe the lattice vibrational phase in the Penrose lattice. Microscopically there is little difference in the lattice vibrations between the Penrose lattice and the regular ones: The bonded atoms vibrate almost in phase at low frequencies and out of phase in the very high-frequency region. Therefore we take the accumulated phase difference as a parameter describing the local vibrational phase difference between vibrating atoms defined by

$$
I_{\lambda}=\sum_{i, j} \sum_{\alpha} u_{i, \lambda}^{\alpha} u_{j, \lambda}^{\alpha}
$$

where the summation is performed over all the bonded atom pairs. Assuming the sinusoidal wave in the regular periodic lattices as $\mathbf{u}_{i, \mathbf{q}}=\mathbf{u}_{0} \cos \left(\mathbf{q} \cdot \mathbf{x}_{i}\right)$, the $I_{\lambda}$ becomes

$$
\begin{aligned}
I_{\lambda} & =\mathbf{u}_{0}^{2} \sum_{i} \cos \left(\mathbf{q} \cdot \mathbf{x}_{i}\right)\left\{\cos \left[\mathbf{q} \cdot\left(\mathbf{x}_{i}+\mathbf{a}\right)\right]+\cos \left[\mathbf{q} \cdot\left(\mathbf{x}_{i}+\mathbf{b}\right)\right]\right\} \\
& =\cos (\mathbf{q} \cdot \mathbf{a})+\cos (\mathbf{q} \cdot \mathbf{b})
\end{aligned}
$$

where $\mathbf{a}$ and $\mathbf{b}$ are the lattice vectors. Then $I_{\lambda}$ is rewritten in terms of the phase differences $q \cdot a$ and $q \cdot b$ between the nearest-neighbor atoms. $I_{\lambda}$ is the multivalued function with respect to the frequency in the anisotropic system and decreases gradually with increasing wave number. The dependence of $I_{\lambda}$ on the boundary conditions appears through the change of the wave vector $q$ in the whole range of frequency.

We show the numerical results of $I_{\lambda}$ for the Penrose lattice with fourth generation in Figs. 9(a) and 9(b). $I_{\lambda}$ decreases with increasing frequency and is not multivalued. Therefore the Penrose lattice may be rather isotropic for the lattice vibrations. This is consistent with the results by the theoretical approach by means of the point group symmetry. Contrary to the regular lattices, the phase shift is found quite clearly below $\omega^{2}=8$ and above that frequency the results remain unchanged for both boundary conditions. Since we have the same critical frequency for the third-generated lattice, this frequency will be characteristic to the Penrose lattice. Above the critical frequency that is independent of the lattice size, we may consider that the lattice vibrations are localized.

Although we have the evidence of the localization above $\omega^{2}=8$, the energy distribution does not necessarily exhibit the concentrations up to $\omega^{2}=14$. In addition, $I_{\lambda}$ yields zero between 8 and 14 and starts decreasing monotonously at $\omega^{2}=14$. To make clear the discrepancy, we plot the averaged local energy per $a$ atom with coordina- tion number $z,\left\langle E\left(l, \omega_{\lambda}\right)\right\rangle_{z}$, versus frequency for the fourth-generated lattice in Fig. 10. Above $\omega^{2}=8$, each case has the frequency regions where the local energy is greater than the local energy averaged over lattice. These regions shift toward the higher frequency range with increasing coordination number. Comparing the $\left\langle E\left(l, \omega_{\lambda}\right)\right\rangle_{z}$ and the density of states, we see there is clear correspondence between the enhancement of local energy and the spectral peaks. So the spectral peaks may be attributed to the localized vibrations of atoms associated with coordination number $z$. Below $\omega^{2}=14$, the only atoms with $z=3$ and 4 vibrate dominantly and contribute to the spectrum. The atoms with $z=3$ and 4 occupy the $59 \%$ of the lattice. Then the participation ratio has the values as large as those at low frequencies. However above that region, the dominantly vibrating atoms with higher coordination numbers, $z=6$ and 7 , are a minority which are only about $7 \%$. Therefore the participation ratios decrease at high frequencies.

From the facts that $P_{\lambda}$ is small and $I_{\lambda}$ is negative above $\omega^{2}=14$, the atoms with definite $z$ associated with
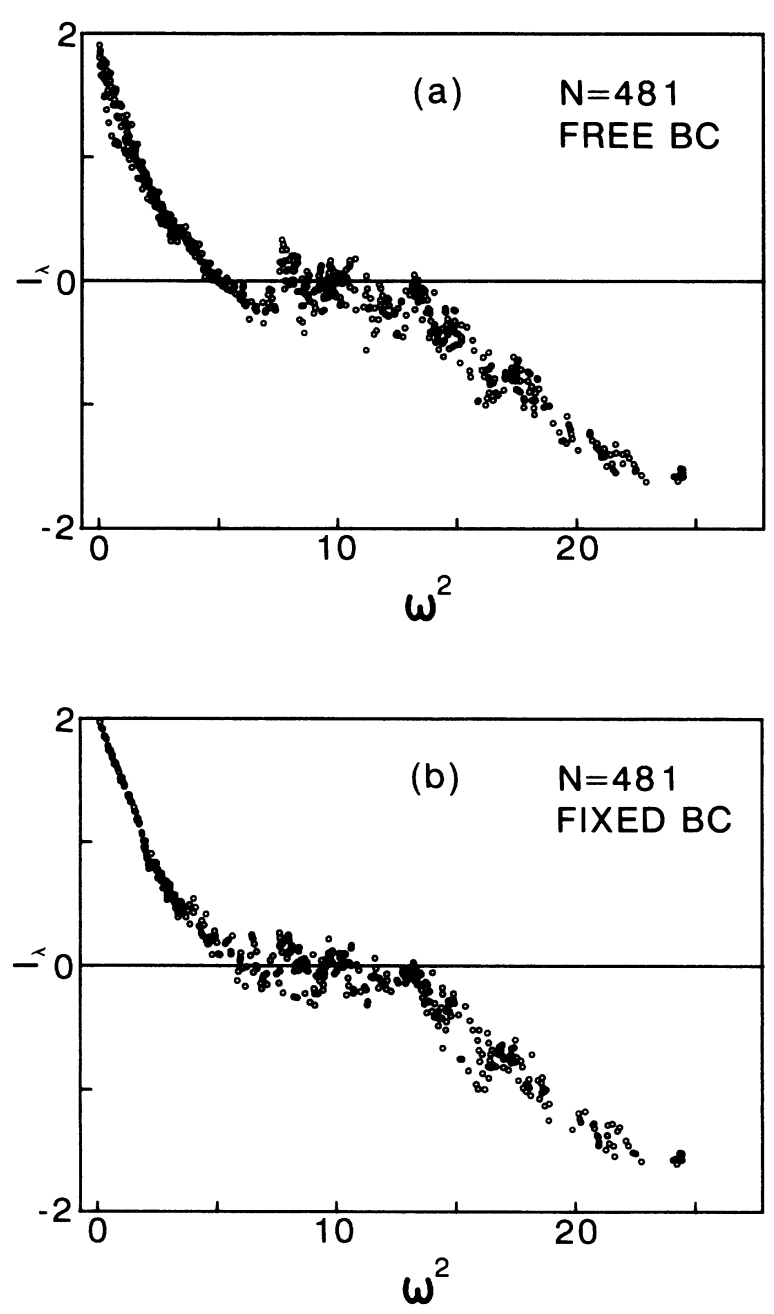

FIG. 9. The accumulated phase difference $I_{\lambda}$ vs squared frequency. (a) Free boundary condition and (b) fixed boundary condition. 


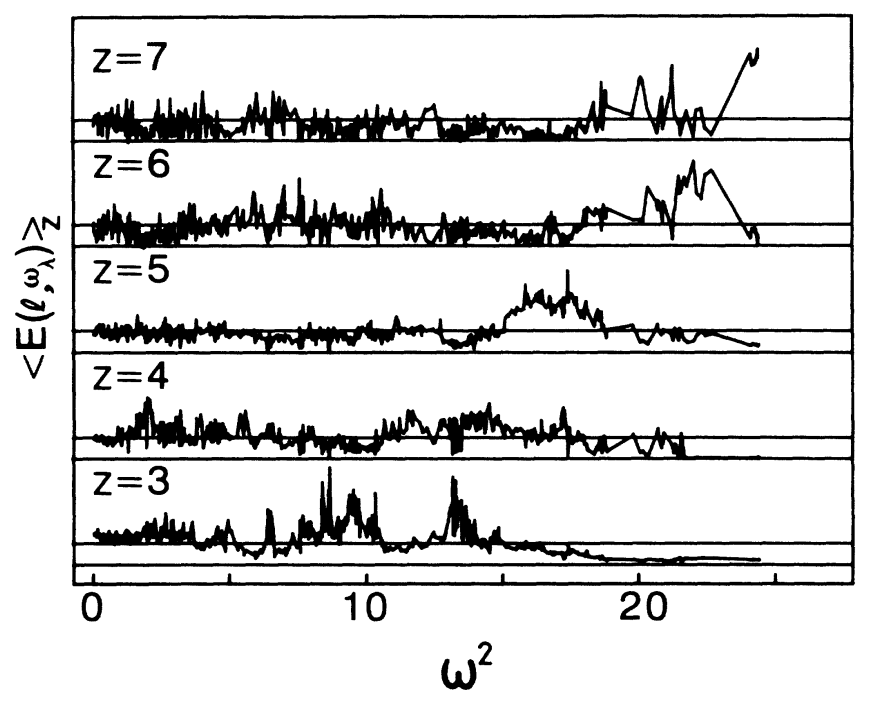

FIG. 10. Local energy spectra with definite coordination number $z$ vs the squared frequency. The lower line of two horizontal lines for each case denotes the zero and the upper the averaged energy over all atoms, respectively.

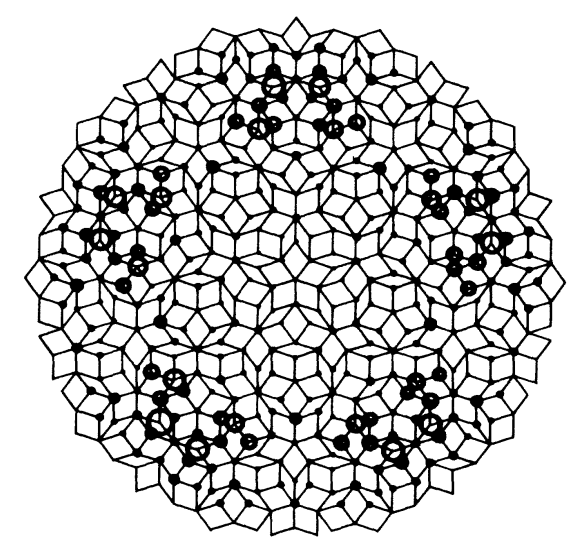

(a)

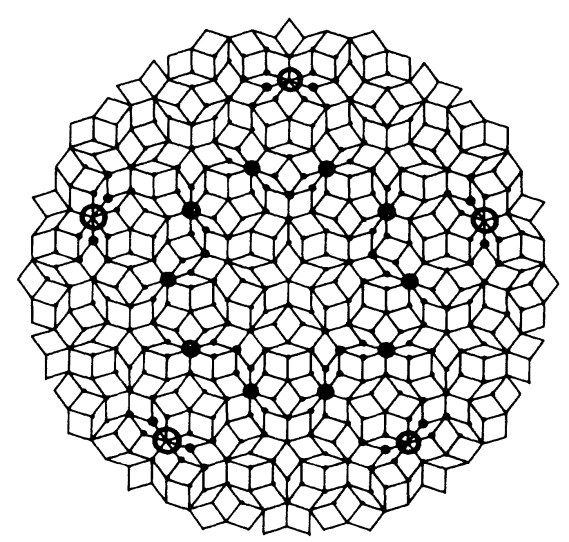

(b)

FIG. 11. Local energy distributions for the Penrose lattice (a) $\omega^{2}=10.46$; (b) $\omega^{2}=24.11$. the spectral peaks have the maximum amplitudes and vibrate in antiphase against the surrounding atoms. This is quite similar to the localized vibrations of the light impurities in a mass-disordered lattice. This similarity is reasonable because the increase of the dynamical matrix elements with respect to the coordination number $z$ has the same effects as the decrease of the corresponding masses, though we set all masses to be unity in our model.

Between $\omega^{2}=8$ and $\omega^{2}=14, I_{\lambda}$ is almost zero and the behavior of $P_{\lambda}$ is also the same as in the frequency region below $\omega^{2}=5$. The vanishing values of $I_{\lambda}$ mean the enlargement of the localized vibrational region. In this frequency region the localization of lattice vibration may be weaker than those in the high-frequency region. The corresponding local energy distributions are shown in Fig. 11 .

Although below $\omega^{2}=8$, the lattice vibrations are influenced by the boundary conditions, they are not extended states since all states are localized in infinite twodimensional systems. ${ }^{16}$ However, we expect that there will be the frequency region with very long localization length and that the concept of phonons may be applicable to the system. If so, we should regard the lattice vibrational states below $\omega^{2}=8$ as weakly localized ones. Therefore the lowest spectral peak in the density of states in this region may be due to van Hove singularity.

\section{SUMMARY AND DISCUSSIONS}

We have numerically studied the lattice vibrations of two-dimensional quasicrystals modeled by Penrose tiling decorated atoms on each vertex. The elastic properties were investigated in the harmonic approximation where the bond stretching and bond bending of the rhombuses are considered to be the potential energy. We set up the equation of motion in terms of the dynamical matrix derived from the elastic potential energy. The vibrational spectra and eigenvectors were obtained by solving the secular equation numerically.

The density of states has several spectral peaks, which are quite similar to those of mass-disordered solids. Since the spectral peaks in the mass-disordered solids are due to van Hove singularities and the localization of the lattice vibrations, there is a possibility that some of the lattice vibrations in the Penrose lattice may be localized. To check the existence of the localization, we examined the frequency spectra, the energy distributions, and the accumulated phase difference under free and fixed boundary conditions. Due to the change of boundary conditions from free to fixed, only the lowest spectral peak shifts to the higher-frequency region. The change in the energy distributions appears in the frequency region below about $\omega^{2}=5$ where the lowest spectral peak appears. The vibrational phase difference between the nearest-neighbor atoms exhibits a critical dependence on the boundary conditions. Below the critical frequency $\omega^{2}=8$, the accumulated phase difference $I_{\lambda}$ is somewhat enhanced in the case of fixed boundary conditions. As this frequency is independent of the lattice size, the lattice vibrations may be localized above the critical frequency where the $I_{\lambda}$ is 
independent of the boundary conditions.

The participation ratio up to $\omega^{2}=14$ remains as large as those at low frequencies and decreases in the highfrequency region. The behavior of the $P_{\lambda}$ with respect to the frequency is due to the decrease of the number of atoms associated with the localized lattice vibrations. The vibrations at frequencies above $\omega^{2}=14$ are quite similar to the localized ones such as the light impurities in the mass-disordered system. Below the frequency $\omega^{2}=14$, a decrease of the $P_{\lambda}$ is not seen, though the lattice vibrations may be still localized in this region. This is because the lattice vibrations concerned with major atoms are localized in a wider region than those above $\omega^{2}=14$. In the one-dimensional Fibonacci lattice, it has been known that self-similar eigenfunctions and the spectral gaps exist as characteristic features. ${ }^{3}$ In the twodimensional case, it would be very interesting if there ex- isted evidence of the self-similarity in the lattice vibrational spectra and also if the spatial variation of the eigenfunctions would be clarified as well as the electronic system. ${ }^{17}$

To conclude, though we expected novel features in the lattice vibrations of Penrose lattice different from those of regular crystals and amorphous solids, the system seems to have the characteristic features rather close to those of disordered systems.

\section{ACKNOWLEDGMENTS}

This work was supported in part by a grant from the Department of Education, Science and Culture of Japan. Numerical calculations were performed by using the facilities at the Hokkaido University Computer Center.
${ }^{1}$ D. Shechtman, I. Blech, D. Gratias, and J. W. Cahn, Phys. Rev. Lett. 53, 1951 (1984).

${ }^{2}$ D. Levine and P. J. Steinhardt, Phys. Rev. Lett. 53, 2477 (1984).

${ }^{3}$ K. Machida and M. Fujita, J. Phys. Soc. Jpn. 55, 1799 (1986).

${ }^{4}$ P. A. Kalugin, A. Yu. Kitaev, and L. S. Levitov, Pis'ma Zh. Eksp. Teor. Fiz. 41, 119 (1985) [JETP Lett. 41, 145 (1985)].

${ }^{5}$ T. C. Lubensky, S. Ramaswamy, and J. Toner, Phys. Rev. B 32, 7444 (1985).

6P. Bak, Phys. Rev. Lett. 54, 1517 (1985); Phys. Rev. B 32, 5764 (1985).

${ }^{7}$ D. Levine, T. C. Lubensky, S. Ostlund, S. Ramaswamy, P. J. Steinhardt, and J. Toner, Phys. Rev. Lett. 54, 1520 (1985).

${ }^{8}$ T. Odagaki and D. Nguyen, Phys. Rev. B 33, 2184 (1986).

${ }^{9}$ M. Kohmoto and B. Sutherland, Phys. Rev. B 34, 3849 (1986).

${ }^{10}$ M. Klenin, Z. Phys. B 65, 1 (1986).

${ }^{11}$ R. Penrose, Bull. Inst. Math. Appl. 10, 266 (1974).
${ }^{12}$ M. Gardner, Sci. Am. 236, 110 (1977).

${ }^{13}$ W. A. Bowers and H. B. Rosenstock, J. Chem. Phys. 18, 1056 (1950). See A. A. Maradudin, E. W. Montroll, and G. H. Weiss, in Solid State Physics, edited by F. Seitz and D. Turnbull (Academic, New York, 1963), Suppl. 3.

${ }^{14}$ P. Dean, Proc. R. Soc. London 260, 263 (1961); P. Dean and M. D. Bacon, ibid. 283, 64 (1965). See also J. Hori, Spectral Properties of Disordered Chains and Lattices (Pergamon, New York, 1968).

${ }^{15}$ D. J. Thouless, Phys. Rep. 13C, 93 (1975); D. C. Licciardello and D. J. Thouless, J. Phys. C 8, 4157 (1975); D. J. Thouless, Phys. Rev. Lett. 39, 1167 (1975).

${ }^{16}$ E. Abraham, P. W. Anderson, D. C. Licciardello, and T. V. Ramakrishnan, Phys. Rev. Lett. 42, 673 (1979); S. John, H. Sompolinsky, and M. J. Stephen, Phys. Rev. B 27, 5592 (1983).

${ }^{17}$ B. Sutherland, Phys. Rev. B 34, 3904 (1986); 35, 9529 (1987). 